\title{
Acquisition of pluripotency in the chick embryo occurs during intrauterine embryonic development via a unique transcriptional network
}

Jae Yong Han ${ }^{1,2^{*}}$, Hyo Gun Lee ${ }^{1}$, Young Hyun Park1, Young Sun Hwang ${ }^{1}$, Sang Kyung Kim', Deivendran Rengaraj ${ }^{3}$, Byung Wook $\mathrm{Cho}^{4}$ and Jeong Mook Lim ${ }^{1}$

\begin{abstract}
Background: Acquisition of pluripotency by transcriptional regulatory factors is an initial developmental event that is required for regulation of cell fate and lineage specification during early embryonic development. The evolutionarily conserved core transcriptional factors regulating the pluripotency network in fishes, amphibians, and mammals have been elucidated. There are also species-specific maternally inherited transcriptional factors and their intricate transcriptional networks important in the acquisition of pluripotency. In avian species, however, the core transcriptional network that governs the acquisition of pluripotency during early embryonic development is not well understood.

Results: We found that chicken NANOG (CNANOG) was expressed in the stages between the pre-ovulatory follicle and oocyte and was continuously detected in Eyal-Giladi and Kochav stage I (EGK.I) to X. However, CPOUV was not expressed during folliculogenesis, but began to be detectable between EGK.V and VI. Unexpectedly, cSOX2 could not be detected during folliculogenesis and intrauterine embryonic development. Instead of CSOX2, cSOX3 was maternally inherited and continuously expressed during chicken intrauterine development. In addition, we found that the pluripotency-related genes such as CENS-1, CKIT, CLIN28A, CMYC, CPRDM14, and CSALL4 began to be dramatically upregulated between EGK.VI and VIII.

Conclusion: These results suggest that chickens have a unique pluripotent circuitry since maternally inherited cNANOG and cSOX3 may play an important role in the initial acquisition of pluripotency. Moreover, the acquisition of pluripotency in chicken embryos occurs at around EGK.VI to VIII.
\end{abstract}

Keywords: Avian, Embryonic development, NANOG, Pluripotency, Transcriptional factor

\section{Background}

The acquisition of pluripotency plays a pivotal role in determining developmental fate during early vertebrate embryogenesis. In mammals, Oct4, Sox2, and Nanog, called core pluripotency transcription factors, act as specific modulators of pluripotency and can control the developmental fate of cells by inhibiting cellular differentiation $[1,2]$. In mice,

\footnotetext{
* Correspondence: jaehan@snu.ac.kr

${ }^{1}$ Department of Agricultural Biotechnology and Research Institute of

Agriculture and Life Sciences, College of Agriculture and Life Sciences, Seoul

National University, Seoul 08826, Korea

${ }^{2}$ Institute for Biomedical Sciences, Shinshu University, Minamiminowa,

Nagano 399-4598, Japan

Full list of author information is available at the end of the article
}

Oct3/4 and Sox2 are maternally inherited transcription factors [3], while Nanog is initially expressed in the compacted morulae [4]. These transcription factors collaborate to constitute a regulatory network, and share many target genes $[5,6]$. They are expressed in every cell during the cleavage period, but are gradually restricted to the inner cell mass. Meanwhile, Oct25 (Pou5f3.2), Oct60 (Pou5f3.3), and Sox3 are maternally inherited to establish the pluripotency network and initiate the maternal to zygotic transition in Xenopus. Since there is no ortholog of Nanog in Xenopus, Ventx plays an important role in pluripotency $[7,8]$. In addition, in zebrafish, Oct4 (Pou5f3), SoxB1, and Nanog are maternally inherited to establish the pluripotency network 
for zygotic genome activation (ZGA) [9, 10]. Although maternally inherited core transcription factors for the initial acquisition and organization of pluripotency are unique to each vertebrate species, their network in vertebrates is well conserved. However, the acquisition of pluripotency and the core pluripotency circuitry during early embryonic development has yet to be investigated in detail in birds. It has been reported that the transition from totipotent state to pluripotent state during early embryonic development seem to be accompanied by the pluripotency regulatory genes under core transcriptional network [11-14]. However, the intricate changes of transcriptional network under regulation of core pluripotency circuitry during the acquisition of pluripotency in avian species are not clear.

After fertilization, chicken embryos undergo a series of developmental events in utero for approximately $24 \mathrm{~h}$, including cellularization, the ZGA and layers increase during the cleavage period, and lineage specification and layer reduction during area pellucida formation [15]. During chicken intrauterine development, the expression of core regulatory genes is spatiotemporally triggered or suppressed under tight transcriptional regulation. Such early developmental pathways, including ZGA, pluripotency acquisition, and lineage segregation, are systematic processes, governed by the concerted action of multiple unknown transcriptional networks [16-18]. In this regard, the core pluripotency transcription factors governing the acquisition of pluripotency with respect to developmental processes during chicken intrauterine development require further investigation. Here, for the first time, we examined the detailed spatiotemporal expression profiles of core pluripotency transcription factors, including chicken NANOG (cNANOG), POUV (cPOUV) and SOXB1 members (cSOX2 and $c S O X 3)$, and determined the developmental stage for the acquisition of pluripotency during intrauterine embryonic development in chicken.

\section{Methods}

\section{Experimental animals and animal care}

The care and experimental use of chickens was approved by the Institute of Laboratory Animal Resources, Seoul National University (SNU-150827-1). Chickens were maintained according to a standard management program at the University Animal Farm, Seoul National University, Korea. The procedures for animal management, reproduction, and embryo manipulation were in adherence with the standard operating protocols of our laboratory.

\section{Alignment and conservation of protein sequences}

In order to identify the percent identities of chicken NANOG, POUV, SOX2, and SOX3 proteins/functional domains with candidate vertebrate species, the NANOG,
POUV, SOX2, and SOX3 amino acid sequences from Gallus gallus, Homo sapiens, Mus musculus, Danio rerio, and Xenopus laevis were aligned with Geneious software version 6.0 (Biomatters, Auckland, New Zealand). Sequence information was obtained from the National Center for Biotechnology Information (NCBI) database (Table 1). All protein sequences were aligned using the Blosum62 scoring matrix, with the gap open penalty set at 12 and the gap extension penalty set at 3 .

\section{Collection of intrauterine eggs, follicles, and oocytes from hens}

The intrauterine embryonic developmental period in the chicken is divided into 10 stages, described and named by Eyal-Giladi and Kochav, and designated EGK.I through to EGK.X [19]. Intrauterine eggs were retrieved from White Leghorn (WL) hens by an abdominal massage technique from our earlier study [17]. Briefly, the abdomen was pushed gently until the shell gland was exposed. The surface of the shell gland expanded when an egg was located there for eggshell formation. After this expansion of the shell gland, the intrauterine egg was gently moved toward the cloaca via massage until it was released. Intrauterine blastoderms were classified according to the criteria of Eyal-Giladi and Kochav [19, 20]. The harvested blastoderms were fixed in $4 \%$ paraformaldehyde in phosphate-buffered saline (PBS) for subsequent experiments. Fertility and abnormalities in the collected blastoderms were determined according to morphology. For the collection of follicles and oocytes, WL hens were sacrificed and ovaries were collected. Follicles were categorized into F1 (30-35 mm), F3 (20-25 mm), F5 (10$15 \mathrm{~mm})$, small yellow follicle $(5-8 \mathrm{~mm})$, and large white follicle (WF, 2-4 mm) [21, 22]. Follicles were dissected to separate theca and granulosa layers and were subsequently homogenized for isolation of RNA after washing with PBS.

Reverse transcription-polymerase chain reaction (RT-PCR) and quantitative real-time PCR (qRT-PCR)

The total RNA of samples was extracted from preovulatory follicles and intrauterine chicken embryos using TRIzol reagent (Invitrogen, Thermo Fisher Scientific, Carlsbad, CA, USA) according to the manufacturer's instructions. The oviposited chicken embryos were classified according to the staging by Hamburger and Hamilton $(\mathrm{HH})$ [23]. From the HH 26-28 embryos, RNA was extracted from chicken embryonic fibroblasts (CEFs) and intact primordial germ cells (PGCs) [24]. The complementary DNA (cDNA) of the sample was synthesized using the Superscript III First-strand Synthesis System (Invitrogen) according to the manufacturer's protocol. The RT-PCR reaction mixture contained $2 \mu \mathrm{L}$ of PCR buffer, $0.5 \mu \mathrm{L}$ of $10 \mathrm{mmol} / \mathrm{L}$ dNTP mixture (Solgent, Daejeon, Korea), 10 pmoles each of forward and reverse primers (Table 2), $1 \mu \mathrm{L}$ 
Table 1 Protein sequence alignment of chicken NANOG, POUV, SOX2, and SOX3 with candidate vertebrate species

\begin{tabular}{llllll}
\hline Protein & Species & Accession no. & Protein length & Percent identities of proteins & Percent identities of functional domains $^{\text {a }}$ \\
\hline cNANOG & Gallus gallus & NP_001139614 & 309 & NA & Homeodomain \\
& Homo sapiens & NP_079141 & 305 & $26.0 \%$ & $64.8 \%$ \\
& Mus musculus & NP_082292 & 305 & $27.5 \%$ & $66.7 \%$ \\
cPOUV & Danio rerio & AEZ64150 & 384 & $20.1 \%$ & $61.1 \%$ \\
& Gallus gallus & NP_001296301 & 389 & NA & Homeodomain \\
& Homo sapiens & NP_002692 & 360 & $36.1 \%$ & $66.7 \%$ \\
& Mus musculus & NP_038661 & 352 & $35.7 \%$ & $66.7 \%$ \\
& Danio rerio & NP_571187 & 472 & $39.5 \%$ & $64.8 \%$ \\
cSOX2 & Xenopus laevis & NP_001081342 & 445 & $37.1 \%$ & $74.1 \%$ \\
& Gallus gallus & AAB09662 & 315 & NA & HMG domain \\
& Homo sapiens & NP_003097 & 317 & $93.4 \%$ & $98.6 \%$ \\
& Mus musculus & NP_035573 & 319 & $92.2 \%$ & $98.6 \%$ \\
& Danio rerio & NP_998283 & 315 & $90.2 \%$ & $97.2 \%$ \\
& Xenopus laevis & NP_001081691 & 311 & $91.1 \%$ & $100 \%$ \\
cSOX3 & Gallus gallus & NP_989526 & 316 & NA & HMG domain \\
& Homo sapiens & NP_005625 & 446 & $69.0 \%$ & $97.2 \%$ \\
& Mus musculus & NP_033263 & 450 & $69.3 \%$ & $97.2 \%$ \\
& Danio rerio & NP_001001811 & 300 & $79.0 \%$ & $95.8 \%$ \\
Xenopus laevis & NP_001007502 & 307 & $82.2 \%$ & $98.6 \%$ \\
\hline
\end{tabular}

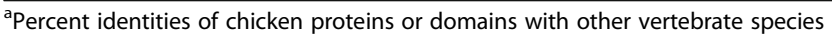

Table 2 Primer sequences used for RT-PCR, in situ hybridization, and qRT-PCR

\begin{tabular}{|c|c|c|c|c|}
\hline Gene & Accession no. & Forward $\left(5^{\prime} \rightarrow 3^{\prime}\right)$ & Reverse $\left(5^{\prime} \rightarrow 3^{\prime}\right)$ & Amplicon size, bp \\
\hline \multicolumn{5}{|c|}{ RT-PCR or in situ hybridization } \\
\hline ¿NANOG & NM_001146142 & CAGCAGACCTCTCCTTGACC & AAGCCCTCATCCTCCACAGC & 586 \\
\hline CPOUV & NM_001309372 & GCCAAGGACCTCAAGCACAA & ATGTCACTGGGATGGGCAGA & 511 \\
\hline $\mathrm{CSOX} 2$ & NM_205188 & CACAACTCGGAGATCAGCAA & GTAGGTAGGCGATCCGTTCA & 471 \\
\hline cSOX3 & NM_204195 & CGGCACCGTACCACTAACTC & GACTCGGAAGCGAACAAAAC & 302 \\
\hline CGAPDH & NM_204305 & CACAGCCACACAGAAGACGG & CCATCAAGTCCACAACACGG & 443 \\
\hline \multicolumn{5}{|l|}{ qRT-PCR } \\
\hline CGAPDH & NM_204305 & ACACAGAAGACGGTGGATGG & GGCAGGTCAGGTCAACAACA & 193 \\
\hline CNANOG & NM_001146142 & CAGCAGACCTCTCCTTGACC & AAAAGTGGGGCGGTGAGATG & 187 \\
\hline CPOUV & NM_001309372 & TGAAGGGAACGCTGGAGAGC & ATGTCACTGGGATGGGCAGAC & 231 \\
\hline cSOX3 & NM_204195 & CGGCACCGTACCACTAACTC & GACTCGGAAGCGAACAAAAC & 302 \\
\hline CENS-1 & NM_001080873 & TGCTCGGCCTTCTGTATCAG & TTCCTCTCGGAACTCCACAG & 181 \\
\hline CTFCP2LI & XM_422087 & TCAGCACATTAAAAGCTGAAAGCA & AGCAATCTCAGTGAGGCACTA & 110 \\
\hline CTBX3 & NM_001270878 & GTGGAAGACGACCCGAAAGT & CACCATCTCCGTGCCTCTTT & 78 \\
\hline CPRDM14 & XM_015282907 & AAATTCCCCTGCCACCTCTG & CCCGCATGTGTTTGTTCAGG & 154 \\
\hline CKIT & NM_204361 & AGCGAACTTCACCTTACCCG & CTGGGAATCCAGTTGCCACA & 181 \\
\hline CLIN28A & NM_001031774 & CCGAGAATGAGTCCCAACCC & GGTGAATTCAACGGCTTCGC & 197 \\
\hline CMYC & NM_001030952 & GAGGAGAACGACAAGAGGCG & CACGCAGGGCAAAGAAACTC & 85 \\
\hline CSALL4 & NM_001080872 & AATTCTGCCAGACGGGGAAG & GCTATGCCATTGCTGAGCAC & 170 \\
\hline
\end{tabular}


of cDNA and 1 IU of Taq DNA polymerase in a $20 \mu \mathrm{L}$ final volume. RT-PCR was performed with an initial incubation at $95^{\circ} \mathrm{C}$ for $10 \mathrm{~min}$, followed by 30 cycles of $95^{\circ} \mathrm{C}$ for $30 \mathrm{~s}$, $60{ }^{\circ} \mathrm{C}$ for $30 \mathrm{~s}$, and $72{ }^{\circ} \mathrm{C}$ for $30 \mathrm{~s}$. PCR was terminated by a final incubation at $72{ }^{\circ} \mathrm{C}$ for 5 min. qRT-PCR was performed using the CFX96 Real-Time PCR Detection System (Bio-Rad, Hercules, CA, USA). The PCR reaction mixture contained $2 \mu \mathrm{L}$ of PCR buffer, $0.5 \mu \mathrm{L}$ of $10 \mathrm{mmol} /$ L dNTP mixture (Solgent), 10 pmoles each of the forward and reverse primers (Table 2), $1 \mu \mathrm{L}$ of $\mathrm{cDNA}, 1 \mu \mathrm{L}$ of EvaGreen (Biotium, Fremont, CA, USA), and 1 IU of Taq DNA polymerase in a $20-\mu \mathrm{L}$ final volume. qRT-PCR was performed with an initial incubation at $95^{\circ} \mathrm{C}$ for $10 \mathrm{~min}$, followed by 40 cycles of $95{ }^{\circ} \mathrm{C}$ for $30 \mathrm{~s}, 60^{\circ} \mathrm{C}$ for $30 \mathrm{~s}$, and $72{ }^{\circ} \mathrm{C}$ for $30 \mathrm{~s}$. The reaction was terminated by a final incubation at the dissociation temperature. The relative gene expression was calculated after normalization with GAPDH and values at stage EGK.X using the formula $2^{-\Delta \Delta C t}$ [25].

\section{In situ hybridization}

To prepare hybridization probes, total RNA from each blastodermal stage was reverse transcribed, and the cDNA was amplified using the primers shown in Table 2. The PCR products of the correct size were cloned with the pGEM-T Easy Vector System (Promega, Madison, WI, USA). After sequence verification, the recombinant plasmids containing the genes of interest were amplified with T7 (T7: 5' -TGTAATACGACTCACTATAGGG-3') and SP6-specific primers (SP6: 5'-CTATTTAGGTGA CACTATAGAAT-3') to prepare the templates for labeling with hybridization probes. Digoxigenin (DIG)-labeled sense and antisense hybridization probes of each gene were transcribed in vitro using the DIG RNA Labeling Kit (Roche Diagnostics, Basel, Switzerland). Whole mount in situ hybridization was performed following the standard protocol for chickens [26, 27]. In addition, intrauterine blastoderms were embedded in paraffin and sectioned at $10 \mu \mathrm{m}$ on a HM $355 \mathrm{~S}$ automatic microtome (Thermo Fisher Scientific). After deparaffinization, rehydration, and antigen retrieval, each slide was mounted with Vectashield Antifade Mounting Medium with DAPI (Vector Laboratories, Burlingame, CA, USA). The embryonic nuclei were evaluated under a Ti-U fluorescence microscope (Nikon, Tokyo, Japan).

\section{Statistical analysis}

All data of qRT-PCR are expressed as mean \pm standard error of mean from three independent experiments. GraphPad Prism software (GraphPad Software, La Jolla, CA, USA) was used to evaluate the data. Significant differences were evaluated by one-way ANOVA with Bonferroni's multiple comparison test between developmental stages. $P<0.05$ was considered statistically significant.

\section{Results}

Expression profiling of core pluripotent transcriptional factors during chicken intrauterine development

To investigate the conservation of transcriptional regulatory networks of pluripotency among vertebrates, initially, we compared protein identities and the conservation of core pluripotent transcription factors, including cPOUV, cSOX2, and cNANOG with human, mouse, zebrafish, and frog. As shown in Table 1, the identities of cNANOG and its homeodomain with the candidate vertebrate species was about $20-26 \%$ and $61-66 \%$, respectively. The identities of cPOUV and its homeodomain with the candidate vertebrate species was about $35-39 \%$ and $64-74 \%$, respectively. Interestingly, the identities of cSOX2 and its high mobility group (HMG) domain with the candidate vertebrate species was about $90-93 \%$ and $97-100 \%$, respectively.

To examine which of the core pluripotent transcription factors are maternally inherited in chicken, $c P O U V$, cNANOG, and $c S O X 2$ genes were evaluated on the stages between WFs and oocytes together with PGC, EGK.X, and CEF samples using RT-PCR. As shown in Fig. 1a, $c N A N O G$ was only expressed during folliculogenesis, indicating that $c N A N O G$ is maternally derived. cPOUV expression was only detected in PGCs and EGK.X embryo, and $c S O X 2$ expression was not detected in any of these samples. To understand the temporal regulation of pluripotency networks during chicken intrauterine development, we examined the expression profiles of core pluripotency transcription factors across developmental stages from the oocyte to the EGK.X embryo (Fig. 1b and c). The results of RT-PCR showed that $c N A N O G$ was detected continuously from the oocyte to stage EGK.X but $c P O U V$ was first detectable at EGK.V and its expression was upregulated thereafter. Unexpectedly, cSOX2 was not expressed during chicken intrauterine stages despite its important function in the pluripotency circuitry (Fig. 1b). The results of qRT-PCR showed highly correlative manner of $c P O U V$ and $c N A$ NOG expressions in the samples examined (Fig. 1c). Taken together, these results suggest that $c N A N O G$ and cPOUV, but not $c S O X 2$, are involved in the acquisition of pluripotency during early development in the chicken.

\section{Cellular localization of CNANOG and CPOUV from the oocyte to stage EGK.X}

To determine the cellular localization and temporal expression of $c N A N O G$ and $c P O U V$, we conducted wholemount in situ hybridization and longitudinal sections over the course of development from EGK.I to EGK.X. As shown in Fig. 2, cNANOG transcripts were rarely detected between EGK.I and EGK.III (Fig. 2a-c and a'-c') and began to be detectable at EGK.IV (Fig. 2d and d'), where they were localized in a heterogeneous manner in the central region of the blastoderm (Fig. 2e and e'), and 
a
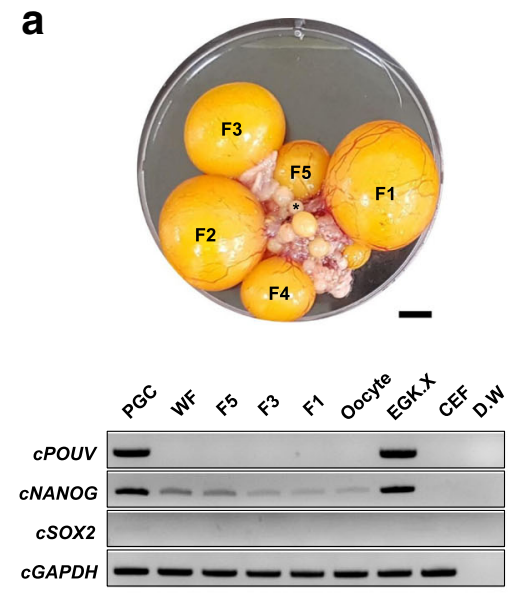

b

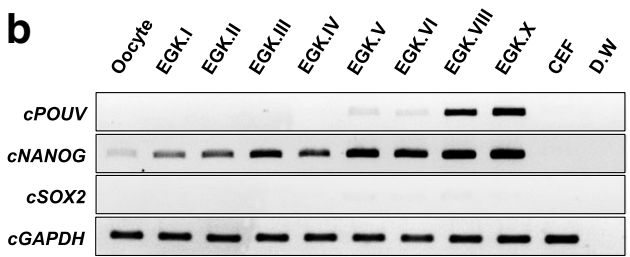

C

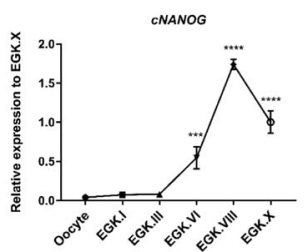

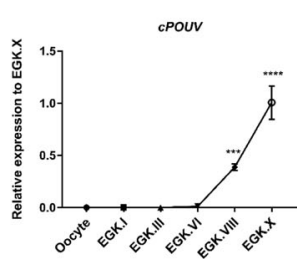

Fig. 1 Core pluripotent transcription factors expression during folliculogenesis and intrauterine embryonic development in chicken. a RT-PCR was conducted to examine the maternally inherited pluripotent transcriptional factors, including CPOUV, CNANOG, and CSOX2 during chick ovarian follicle development. Follicles in the ovary are indicated by hierarchical follicle (F1 to F5) and a representative white follicle is indicated by an asterisk. b RT-PCR was conducted to examine expression profiling of CPOUV, CNANOG, and CSOX2 from the oocyte to EGK.X. CEF was used as a negative control for both A and B. c The CPOUV and CNANOG gene expression in oocyte and intrauterine chicken embryos relative to EGK.X was analyzed using qRT-PCR. cGAPDH was used as a reference gene. Results are shown as mean \pm standard error of mean $(n=3)$. Significant differences of the relative gene expression between consecutive developmental stages (Oocyte vs. EGK.I, EGK.I vs. EGK.III, EGK.III vs. EGK.VI, EGK.VI vs. EGK.VIII, and EGK.VIII vs. EGK.X) were represented as *** $P<0.001$ and ${ }^{* * *} P<0.0001$. Scale bar $=1 \mathrm{~cm}$

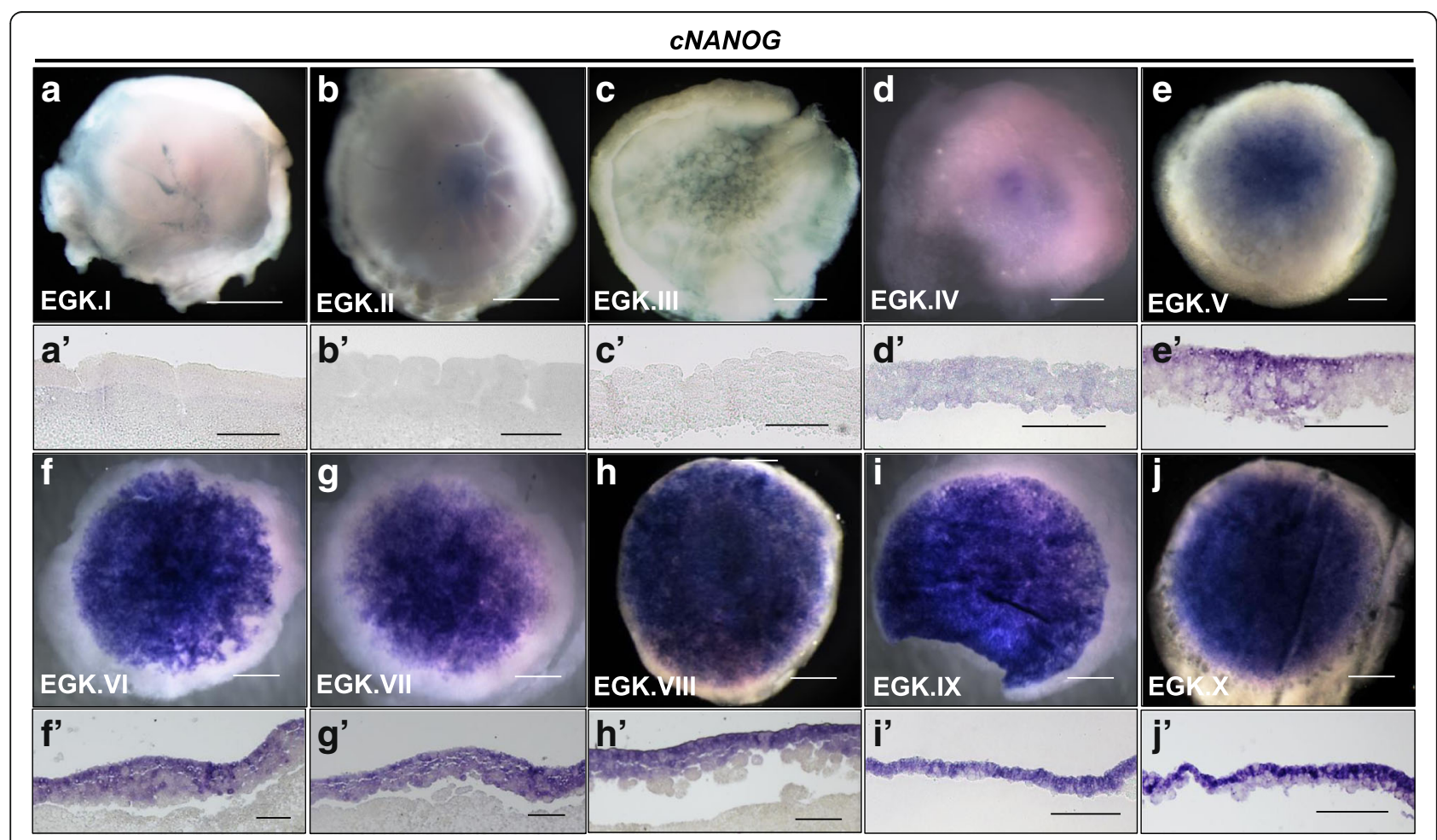

Fig. 2 Cellular localization of CNANOG during intrauterine development of the chicken embryo. In situ hybridization was performed on the whole-mount $(\mathbf{a}-\mathbf{j})$ and longitudinal sections $\left(\mathbf{a}^{\prime}-\mathbf{j}^{\prime}\right)$ of intrauterine chicken embryos to detect cellular localization of $c$ NANOG. Scale bars $=1 \mathrm{~mm}$ $(\mathbf{a}-\mathbf{j})$ and $200 \mu \mathrm{m}\left(\mathbf{a}^{\prime}-\mathbf{j}^{\prime}\right)$ 
the intensity remarkably increased at stage EGK.VI (Fig. $2 \mathrm{f}$ and $\mathrm{f}^{\prime}$ ). During the period of area pellucida formation (EGK.VII-EGK.X), the cNANOG transcripts increased, and were localized to the upper layer of the blastoderm (Fig. 2g-j and $\mathrm{g}^{\prime}-\mathrm{j}$ '). At EGK.X, cNANOG transcripts were exclusively expressed in the epiblast region (Fig. 2j and j'). Meanwhile, cPOUV transcripts were not detected during the EGK.I-VI stages, at which point there is a period of cell layer increase (Fig. 3a-f and a'-f'). cPOUV transcripts started to be detected at EGK.VII and were clearly expressed in a salt-and-pepper manner in the blastoderm before EGK.X (Fig. 3g-j and g'-j'). At EGK.X, cPOUV transcripts were evenly expressed in the upper layer, called the epiblast, or expressed in a heterogeneous manner in the lower layer, called the hypoblast (Fig. 3j').

\section{Expression profiling of $\mathrm{CSOX} 2$ and $\mathrm{CSOX} 3$ during intrauterine development}

Since cSOX2 was not detected in any of the samples tested by RT-PCR (Fig. 1), we further examined whether cSOX2 is expressed during early embryonic development in chicken using whole-mount in situ hybridization. As shown in Fig. 4, cSOX2 was not detected at the preovipositional stages of the chicken embryo (Fig. 4a). Meanwhile, we confirmed that $c S O X 2$ was strongly expressed in the primitive streak at $\mathrm{HH}$ stages 6 and 8
(Fig. $4 \mathrm{~b}$ and c). In addition, we examined the expression profiling of $c S O X 3$ (another member of SOXB1 family) during selective intrauterine development using RT-PCR and qRT-PCR. As shown in Fig. 4d, cSOX3 was maternally inherited and continuously detected from the oocyte to the EGK.X embryo. As determined by qRT-PCR, cSOX3 expression was sharply elevated after EGK.III (Fig. 4e). When we examine the identities of cSOX3 and its HMG domain with the candidate vertebrate species, it shows about $69-82 \%$ and $95-98 \%$ identities, respectively (Table 1). Moreover, similar to cSOX2, cSOX1 expression was not detected in any stages during intrauterine development (data not shown). Collectively, these results imply that the process of pluripotency acquisition in chickens may be initiated by $c N A N O G$ and cSOX3 ahead of $c P O U V$ without $c S O X 2$.

\section{Pluripotency-related marker expression during chicken intrauterine development}

To further investigate the developmental stage in the acquisition of pluripotency and identify the factors involved in the chicken pluripotency network, we examined the comprehensive pluripotency-related marker expression by qRT-PCR across developmental stages from the oocyte to the EGK.X embryo. First, we examined the expression profile of cENS-1, which is a

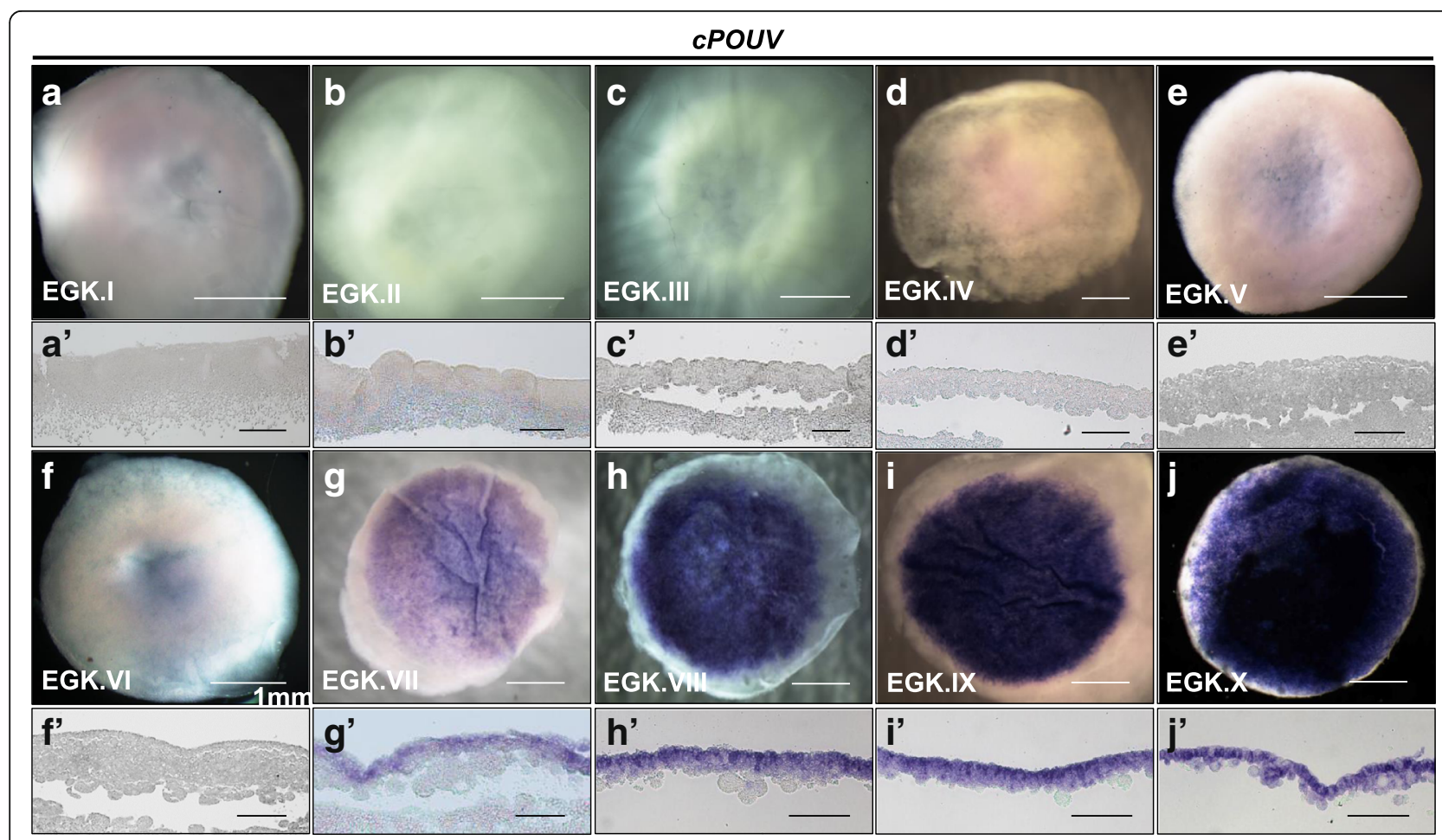

Fig. 3 Cellular localization of CPOUV during intrauterine development of the chicken embryo. In situ hybridization was performed on the wholemount $(\mathbf{a}-\mathbf{j})$ and longitudinal sections $\left(\mathbf{a}^{\prime}-\mathbf{j}^{\prime}\right)$ of intrauterine chicken embryos to detect cellular localization of $c P O U V$. Scale bars $=1 \mathrm{~mm}(\mathbf{a}-\mathbf{j})$ and $200 \mu \mathrm{m}\left(\mathbf{a}^{\prime}-\mathbf{j}^{\prime}\right)$ 
a

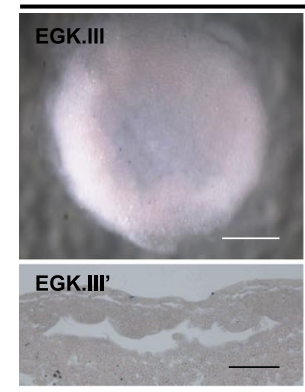

b

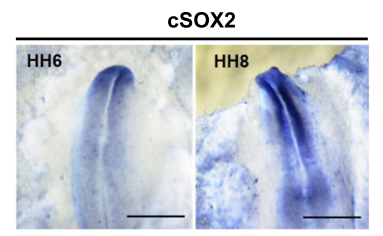

d

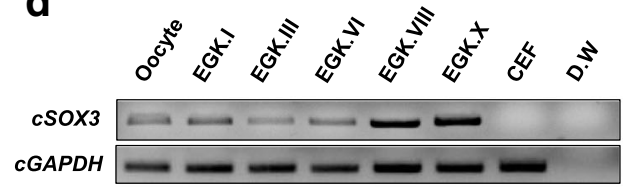

cSOX2
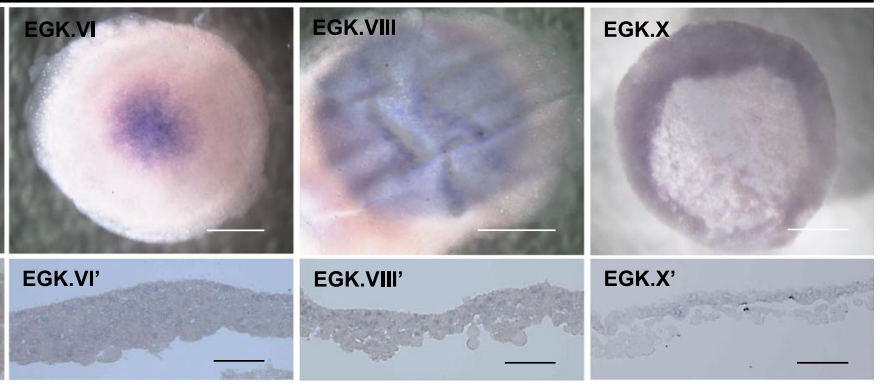

C

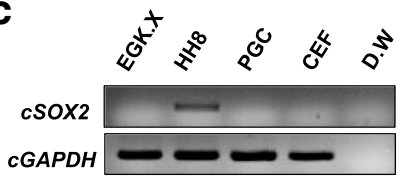

e

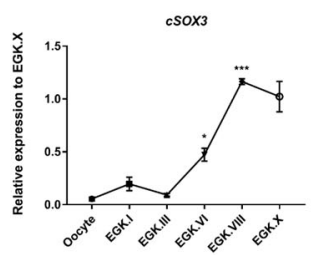

Fig. 4 Expression profiling of $\mathrm{cSOX}_{2}$ and $\mathrm{cSOX} 3$ during chicken embryonic development. a In situ hybridization was performed on the whole-mount and longitudinal sections in pre-oviposition stages of chick blastoderm (EGK.III, EGK.VIII and EGK.X) to detect cSOX2 transcripts. b cSOX2 transcripts are clearly detected at neural induction stages between $\mathrm{HH} 6$ and 8 by whole-mount in situ hybridization. c RT-PCR was conducted to confirm cSOX2 expression in EGK.X, HH8, PGC, and CEF. $\mathbf{d}$ RT-PCR was conducted to examine cSOX3 expression from the oocyte to the EGK.X embryo. CEF was used as a negative control. e The CSOX3 gene expression in oocyte and intrauterine chicken embryos relative to EGK.X was analyzed using qRT-PCR. CGAPDH was used as a reference gene. Results are shown as mean \pm standard error of mean $(n=3)$. Significant differences of the relative gene expression between consecutive developmental stages (Oocyte vs. EGK.I, EGK.I vs. EGK.III, EGK.III vs. EGK.VI, EGK.VI vs. EGK.VIII, and EGK.VIII vs. EGK.X) were represented as ${ }^{*} P<0.05$ and ${ }^{* *} P<0.001$. Scale bars $=1 \mathrm{~mm}$ (whole-mount of embryo) and $200 \mu \mathrm{m}$ (longitudinal sections of embryo)

restrictive chicken endogenous retrovirus-like sequence and embryonic stem cell marker [28]. We found that cENS-1 is significantly upregulated between EGK.VI and VIII but its expression is significantly downregulated between EGK.VIII and $\mathrm{X}$, which is similar to the expression of $c N A N O G$ during developmental stages (Fig. 5a). Next, we investigated the expression of naive pluripotency-related markers, including $C T F C P 2 L 1, C T B X 3$, and $c P R D M 14$, and also general pluripotency-related markers, including $c K I T$, cLIN28A, cMYC, and CSALL4 [2, 29, 30]. These genes have been mainly defined in the mammalian species, however their role on the pluripotency acquisition during chicken intrauterine development is not clear. In our results, the naive pluripotency markers $c T F C P 2 L 1$ and $c T B X 3$ were found to be maternally inherited genes, and expression of the $C P R D M 14$ gene was gradually upregulated from EGK.VI until EGK.X (Fig. 5b). The general pluripotency markers, including $c L I N 28 A, c M Y C$, and $c S A L L 4$ were significantly upregulated between EGK.VI and VIII, and the expression of $c K I T$ was upregulated between EGK.VIII and $\mathrm{X}$ (Fig. 5c). Taken together, these results suggest that the acquisition of pluripotency during chick embryonic development occurs at around stage EGK.VI to EGK.VIII.

\section{Discussion}

The transition to the pluripotent state from the totipotent state in embryonic development is necessary for ZGA, cell cleavage, and regulation of cell fate [31-33]. In early embryogenesis, several transcription factors play a pivotal role in pluripotent acquisition and maintenance during embryogenesis. To date, the mechanisms of acquisition of pluripotency have been intensively studied in mammals and several vertebrates in vivo and in vitro. Regulation of Nanog is important for early development and the acquisition of pluripotency in the epiblast in mammals [34]. Pouff1 is expressed as early as the preimplantation embryo in the mouse [34], and also the mouse pluripotent embryonic stem cells (ESCs) are controlled by Pou $5 f 1$. Another core transcription factor, Sox2, which belongs to the SoxB1 subfamily of genes [35], is also essential for the maintenance of the undifferentiated state in ESCs [36, 37]. 


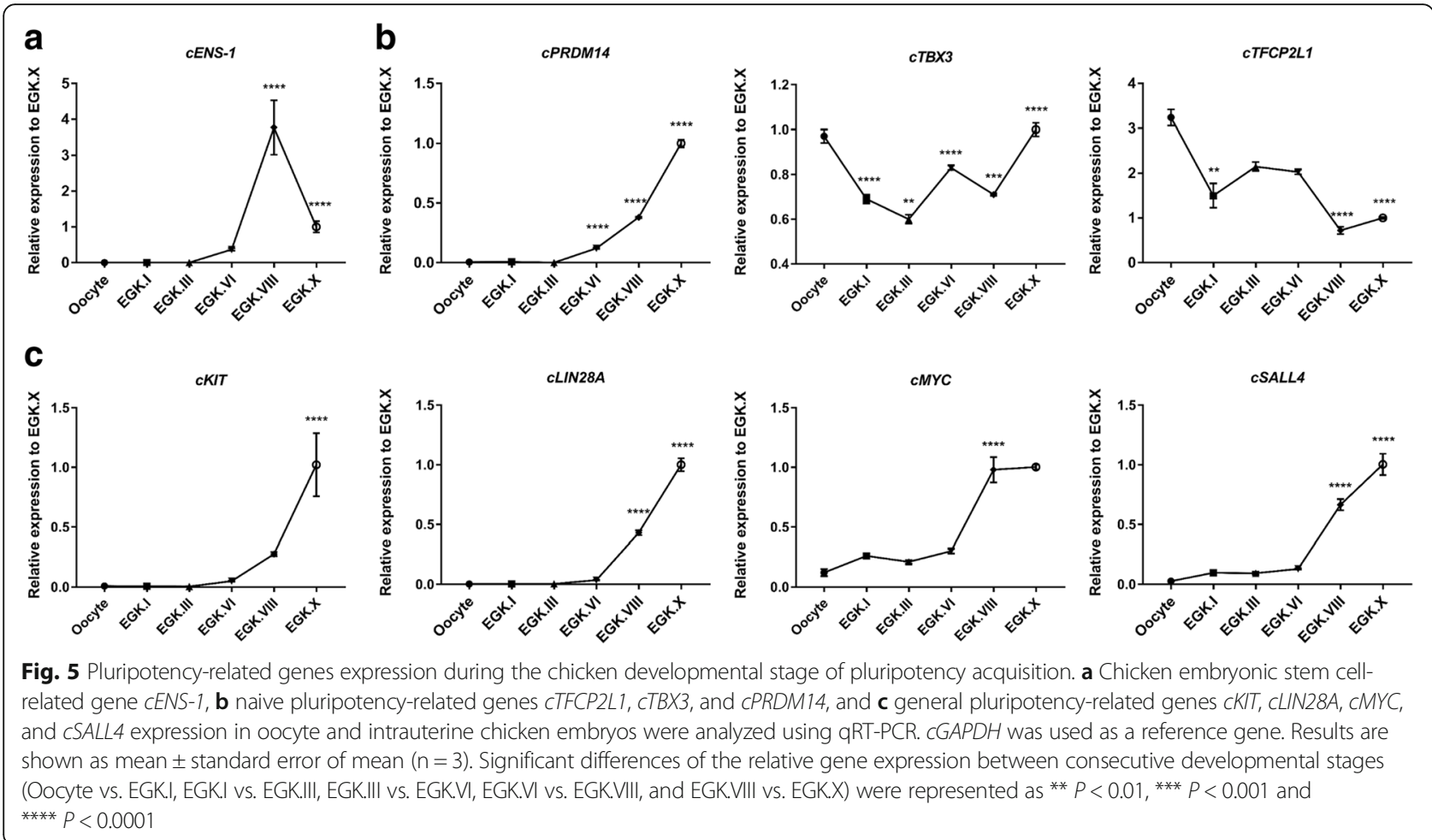

In this regards, transcriptional factors, including Nanog, Pou5f1 and Sox 2 play pivotal role in core regulatory network of pluripotency. Our results showed that the protein alignment of these core pluripotent transcription factors in chicken with other vertebrates revealed that they are fairly well conserved in the protein sequence among vertebrates. In particular, SOX2 has a sequence similarity of more than $90 \%$ in human, mouse, chicken, zebrafish, and African clawed frog. This may imply that these core transcriptional factors share the similar role in the pluripotency network among vertebrates. Although there are only a few studies, it has been recently reported that pluripotency seems to be evolutionarily conserved among amniotes, and mammalian core transcriptional factors could reprogram non-mammalian somatic cells into pluripotent stem cells $[30,38,39]$.

However, the core pluripotent transcription factors that govern the acquisition of pluripotency during preovipositional development have yet to be investigated in birds. Although both PouSf1 and Sox 2 are maternally inherited transcripts in mouse and they induce Nanog expression before ZGA [40], according to our results, the chicken seems to have a distinctive process for the acquisition of pluripotency. In this report, $c S O X 2$ is not expressed during folliculogenesis and intrauterine embryonic development, but detected in the embryo after oviposition, indicating that it is not involved in the initial acquisition of pluripotency. This is consistent with a previous report that cSOX2 in the oviposited chicken embryo is first detected from $\mathrm{HH}$ stage 4 as the earliest panneural marker in the specified neuroectoderm $[41,42]$. Thus, unlike in mammals, $c S O X 2$ in chicken seems to only be involved in early neural specification without a role in pluripotency networks. In the case of lower vertebrates, Sox $19 b$ is maternally inherited in Danio rerio among the SoxB1 family and plays an important role in the acquisition of pluripotency, whereas Sox3 carries out such a maternal contribution in Xenopus laevis [10, 43]. In avian species, it was recently reported that both finch and chick blastoderms at oviposition remarkably expressed SOX3 [30], which is also known to be expressed in epiblast precursors [42]. Since the SOXB1 factors share more than $90 \%$ amino acid identity in the DNA binding HMG box region for transcriptional activation [35], acquisition of pluripotency in avian species may be regulated by another chicken SOXB1 family member instead of $c S O X 2$. Our results show that $c S O X 3$ may play important role in pluripotency network instead of $c S O X 2$ in avian species. It has been reported that mammalian SOX3 can replace the function of SOX2 during the reprogramming process, and SOX3 can compensate the absence of SOX2 to maintain the pluripotency and self-renew of ESC [44-47]. Similar to the pattern of $c N A N O G$ expression, intriguingly, maternally inherited ${ }^{2 S O X 3}$ is upregulated between EGK.III and EGK.VI, indicating that cSOX3 may involve in the initial establishment of pluripotency network in 
chicken embryos. Accordingly, further detailed investigation is required to determine how SOX3 is involved and regulated in the pluripotency network in avian species.

Furthermore, $c N A N O G$ transcripts were detected from between the white follicle and oocyte stages, indicating that among the core transcriptional factors, $c N A N O G$ is maternally inherited in embryos. cNANOG transcripts were weakly expressed compared with the EGK.X embryo, but its expression was dramatically increased between EGK.V and VI and localized in a heterogeneous manner in the central region of chick embryos. $c P O U V$ was not detected until EGK. VI, however, its expression was dramatically increased between EGK VI and VIII in this study. Therefore, $c N A N O G$ and $c S O X 3$ seem to be regulated independently from $c P O U V$ and play an important role in the initial pluripotency network prior to $c P O U V$ during early embryonic development in chicken.

To understand a comprehensive pluripotency network during chick early embryonic development, we compared the relative expression of pluripotency-related genes in embryos from oocyte to EGK.X. Among the naive pluripotency markers, including $c T F C P 2 L 1, c P R D M 14$, and $c T B X 3$ $[13,48,49]$, both $c T F C P 2 L 1$ and $c T B X 3$ seem to be maternally supplied while expression of $C P R D M 14$ was significantly increased between EGK.III and VI. It is known that TFCP2L upregulates NANOG via LIF-independent pathways and TBX3 is directly bound at NANOG and functions in upregulation of NANOG in mammals [50, 51]. In this regard, maternally inherited $c T F C P 2 L 1$ and $c T B X 3$ may regulate the initial upregulation of $c N A N O G$ or may be involved in the initial acquisition of pluripotency in chicken. Meanwhile, chicken ESC marker $c E N S-1$ and the general pluripotency markers $c K I T$ and $c L I N 28 A$ were gradually upregulated from EGK.VI until EGK.X, whereas $c M Y C$ and cSALL4 seem to be maternally inherited but also dramatically upregulated between EGK.VI and VIII. Taken together, most of the pluripotency-associated genes were remarkably upregulated between EGK.VI and VIII, indicating that acquisition of the pluripotency network in the chicken embryo may be established between EGK.VI and VIII.

Meanwhile, it has been reported that the ZGA may be accompanied by acquisition of pluripotency via transcriptional factors in vertebrates $[9,52,53]$. Especially, in the frogs and zebrafish, acquisition of pluripotency is associated with ZGA, whereas acquisition of pluripotency occurred after ZGA in mice [39]. Although ZGA in avian species has not been identified yet, it was reported that the RNA polymerase II started to be activated during the late EGK.II to early EGK.III in chicken [16]. Accordingly, the understanding of the acquisition of pluripotency association with ZGA and the intricate molecular mechanisms of pluripotency regulating chicken embryo development requires further investigation.

\section{Conclusion}

In conclusion, we found that among the core pluripotent transcription factors, $c N A N O G$ was maternally inherited and continuously expressed, but $c P O U V$ was significantly upregulated between EGK.VI and VIII, and $c S O X 3$ instead of $c S O X 2$ was maternally inherited and continuously detected during intrauterine embryonic development in the chicken. Furthermore, we showed that the acquisition of pluripotency in the chick embryo may actively occurs at around stage EGK.VI to EGK.VIII, and birds seem to have a distinct regulatory mechanism of pluripotency compared with other vertebrates. Further studies should focus on the detailed mechanism of the pluripotency network via functional validation of transcriptional factors during early development in avian species from an evo-devo perspective.

\section{Abbreviations}

CEF: Chicken embryonic fibroblast; EGK: Eyal-Giladi and Kochav stage; ENS1: Embryonic normal stem cell 1; GADPH: Glyceraldehyde 3-phosphate dehydrogenase; HH: Hamburger Hamilton stage; HMG: High mobility group; iPSC: Induced pluripotent stem cell; KIT: KIT proto-oncogene receptor tyrosine kinase; LIN28A: Lin-28 homolog A; MYC: MYC proto-oncogene bHLH transcription factor; NANOG: Nanog homeobox; NCBI: National Center for Biotechnology Information; PGC: Primordial germ cell; Pou5f1: POU domain class 5 transcription factor 1; Pou5f3: POU domain class 5 transcription factor 3; POUV: POU domain class 5 transcription factor 3; PRDM14: PR/SET domain 14; qRT-PCR: Quantitative realtime-polymerase chain reaction; RT-PCR: Reverse transcription-polymerase chain reaction; SALL4: Spalt like transcription factor 4 ; SOX2: SRY-box 2; SOX3: SRY-box 3; TBX3: T-box 3; TFCP2L1: Transcription factor CP2-like 1; WF: Large white follicle; ZGA: Zygotic genome activation

\section{Acknowledgements}

Not applicable.

\section{Funding}

This work was supported by the National Research Foundation of Korea (NRF) grant funded by the Korea government (MSIP) (No.2015R1A3A2033826) and the International Research \& Development Program of the National Research Foundation of Korea (NRF) funded by the Ministry of Science, ICT and Future Planning of Korea (NRF-2016K1A3A1A21005676).

\section{Availability of data and materials}

The datasets during and/or analyzed during the current study available from the corresponding authors on reasonable request.

\section{Authors' contributions}

JYH and HGL designed the research. HGL, YHP, SKK carried out and analyzed the experiments. JYH, YSH, BWC and JML interpreted and critically reviewed the data. JYH, YHP, YSH and DR wrote the manuscript. All authors have read and approved the final manuscript.

\section{Ethics approval}

The care and experimental use of chickens were approved by the Institute of Laboratory Animal Resources, Seoul National University.

Consent for publication

Not applicable.

\section{Competing interests}

The authors declare that they have no competing interests.

\section{Author details}

${ }^{1}$ Department of Agricultural Biotechnology and Research Institute of Agriculture and Life Sciences, College of Agriculture and Life Sciences, Seoul National University, Seoul 08826, Korea. ${ }^{2}$ Institute for Biomedical Sciences, 
Shinshu University, Minamiminowa, Nagano 399-4598, Japan. ${ }^{3}$ Department of Animal Science and Technology, Chung-Ang University, Anseong, Gyeonggi-do 17546, Korea. ${ }^{4}$ Department of Animal Science, College of Natural Resources and Life Sciences, Pusan National University, Miryang 50463, Korea.

\section{Received: 11 September 2017 Accepted: 26 February 2018} Published online: 10 April 2018

\section{References}

1. Orkin SH, Wang J, Kim J, Chu J, Rao S, Theunissen TW, et al. The transcriptional network controlling pluripotency in ES cells. Cold Spring Harb Symp Quant Biol. 2008;73:195-202.

2. Yeo JC, $\mathrm{Ng} \mathrm{HH}$. The transcriptional regulation of pluripotency. Cell Res. 2013; 23:20-32.

3. Pesce M, Wang $X$, Wolgemuth DJ, Scholer $H$. Differential expression of the Oct-4 transcription factor during mouse germ cell differentiation. Mech Dev. 1998:71:89-98

4. Chambers I, Colby D, Robertson M, Nichols J, Lee S, Tweedie S, et al. Functional expression cloning of Nanog, a pluripotency sustaining factor in embryonic stem cells. Cell. 2003;113:643-55.

5. Boyer LA, Lee TI, Cole MF, Johnstone SE, Levine SS, Zucker JP, et al. Core transcriptional regulatory circuitry in human embryonic stem cells. Cell. 2005;122:947-56.

6. Loh YH, Wu Q, Chew JL, Vega VB, Zhang W, Chen X, et al. The Oct4 and Nanog transcription network regulates pluripotency in mouse embryonic stem cells. Nat Genet. 2006;38:431-40.

7. Hinkley CS, Martin JF, Leibham D, Perry M. Sequential expression of multiple POU proteins during amphibian early development. Mol Cell Biol. 1992;12: 638-49.

8. Yang J, Aguero T, King ML. The xenopus maternal-to-zygotic transition from the perspective of the germline. Curr Top Dev Biol. 2015;113:271-303.

9. Lee MT, Bonneau AR, Takacs CM, Bazzini AA, DiVito KR, Fleming ES, et al. Nanog, Pou5f1 and SoxB1 activate zygotic gene expression during the maternal-to-zygotic transition. Nature. 2013;503:360-4.

10. Okuda Y, Yoda H, Uchikawa M, Furutani-Seiki M, Takeda H, Kondoh H, et al. Comparative genomic and expression analysis of group B1 sox genes in zebrafish indicates their diversification during vertebrate evolution. Dev Dyn. 2006:235:811-25

11. Munoz Descalzo S, Rue P, Garcia-Ojalvo J, Martinez AA. Correlations between the levels of Oct4 and Nanog as a signature for naive pluripotency in mouse embryonic stem cells. Stem Cells. 2012;30:2683-91.

12. Boroviak T, Loos R, Bertone P, Smith A, Nichols J. The ability of inner-cellmass cells to self-renew as embryonic stem cells is acquired following epiblast specification. Nat Cell Biol. 2014;16:516-28.

13. Nichols J, Smith A. Naive and primed pluripotent states. Cell Stem Cell. 2009:4:487-92.

14. Deb A, Sarkar A, Ghosh Z. Dissecting the variation in transcriptional circuits between naive and primed pluripotent states. FEBS Lett. 2017;591:2362-75.

15. Sheng G. Day-1 chick development. Dev Dyn. 2014;243:357-67.

16. Nagai $H$, Sezaki M, Kakiguchi K, Nakaya $Y$, Lee HC, Ladher $R$, et al. Cellular analysis of cleavage-stage chick embryos reveals hidden conservation in vertebrate early development. Development. 2015;142:1279-86.

17. Lee HC, Choi HJ, Park TS, Lee SI, Kim YM, Rengaraj D, et al. Cleavage events and sperm dynamics in chick intrauterine embryos. PLoS One. 2013:8:e80631.

18. Lee HC, Choi HJ, Lee HG, Lim JM, Ono T, Han JY. DAZL expression explains origin and central formation of primordial germ cells in chickens. Stem Cells Dev. 2016:25:68-79.

19. Eyal-Giladi H, Kochav S. From cleavage to primitive streak formation: a complementary normal table and a new look at the first stages of the development of the chick. I. General morphology. Dev Biol. 1976;49:321-37.

20. Kochav S, Ginsburg M, Eyal-Giladi H. From cleavage to primitive streak formation: a complementary normal table and a new look at the first stages of the development of the chick. II. Microscopic anatomy and cell population dynamics. Dev Biol. 1980;79:296-308.

21. Gilbert $A B$, Perry MM, Waddington $D$, Hardie MA. Role of atresia in establishing the follicular hierarchy in the ovary of the domestic hen (Gallus domesticus). J Reprod Fertil. 1983;69:221-7.

22. Lovell TM, Gladwell RT, Groome NP, Knight PG. Ovarian follicle development in the laying hen is accompanied by divergent changes in inhibin A, inhibin
$B$, activin $A$ and follistatin production in granulosa and theca layers. J Endocrinol. 2003;177:45-55.

23. Hamburger $\mathrm{V}$, Hamilton $\mathrm{HL}$. A series of normal stages in the development of the chick embryo. J Morphol. 1951;88:49-\&

24. Kim JN, Kim MA, Park TS, Kim DK, Park HJ, Ono T, et al. Enriched gonadal migration of donor-derived gonadal primordial germ cells by immunomagnetic cell sorting in birds. Mol Reprod Dev. 2004;68:81-7.

25. Livak KJ, Schmittgen TD. Analysis of relative gene expression data using real-time quantitative PCR and the 2(-Delta Delta C(T)) Method. Methods. 2001;25:402-8.

26. Stern CD. Detection of multiple gene products simultaneously by in situ hybridization and immunohistochemistry in whole mounts of avian embryos. Curr Top Dev Biol. 1998;36:223-43.

27. Rengaraj D, Kim DK, Zheng YH, Lee SI, Kim H, Han JY. Testis-specific novel transcripts in chicken: in situ localization and expression pattern profiling during sexual development. Biol Reprod. 2008;79:413-20.

28. Acloque H, Risson V, Birot AM, Kunita R, Pain B, Samarut J. Identification of a new gene family specifically expressed in chicken embryonic stem cells and early embryo. Mech Dev. 2001;103:79-91.

29. Zhang J, Tam WL, Tong GQ, Wu Q, Chan HY, Soh BS, et al. Sall4 modulates embryonic stem cell pluripotency and early embryonic development by the transcriptional regulation of Pou5f1. Nat Cell Biol. 2006;8:1114-23.

30. Mak SS, Alev C, Nagai H, Wrabel A, Matsuoka Y, Honda A, et al. Characterization of the finch embryo supports evolutionary conservation of the naive stage of development in amniotes. elife. 2015;4:e07178.

31. Kidder GM, McLachlin JR. Timing of transcription and protein synthesis underlying morphogenesis in preimplantation mouse embryos. Dev Biol. 1985;112:265-75.

32. Camous S, Kopecny V, Flechon JE. Autoradiographic detection of the earliest stage of $[3 \mathrm{H}]$-uridine incorporation into the cow embryo. Biol Cell. 1986:58:195-200.

33. Jarrell VL, Day BN, Prather RS. The transition from maternal to zygotic control of development occurs during the 4-cell stage in the domestic pig, Sus scrofa: quantitative and qualitative aspects of protein synthesis. Biol Reprod. 1991;44:62-8.

34. Scholer HR. Octamania: the POU factors in murine development. Trends Genet. 1991;7:323-9.

35. Miyagi $\mathrm{S}$, Kato $\mathrm{H}$, Okuda $\mathrm{A}$. Role of SoxB1 transcription factors in development. Cell Mol Life Sci. 2009;66:3675-84.

36. Wegner M. From head to toes: the multiple facets of Sox proteins. Nucleic Acids Res. 1999;27:1409-20.

37. Wood HB, Episkopou V. Comparative expression of the mouse Sox1, Sox2 and Sox3 genes from pre-gastrulation to early somite stages. Mech Dev. 1999;86:197-201.

38. Rossello RA, Chen CC, Dai R, Howard JT, Hochgeschwender U, Jarvis ED. Mammalian genes induce partially reprogrammed pluripotent stem cells in non-mammalian vertebrate and invertebrate species. elife. 2013:2:e00036.

39. Paranjpe SS, Veenstra GJ. Establishing pluripotency in early development. Biochim Biophys Acta. 2015:1849:626-36.

40. Rodda DJ, Chew JL, Lim LH, Loh YH, Wang B, Ng HH, et al. Transcriptional regulation of nanog by OCT4 and SOX2. J Biol Chem. 2005;280:24731-7.

41. Streit A, Sockanathan S, Perez L, Rex M, Scotting PJ, Sharpe PT, et al. Preventing the loss of competence for neural induction: HGF/SF, L5 and Sox-2. Development. 1997;124:1191-202.

42. Rex M, Orme A, Uwanogho D, Tointon K, Wigmore PM, Sharpe PT, et al. Dynamic expression of chicken Sox2 and Sox3 genes in ectoderm induced to form neural tissue. Dev Dyn. 1997;209:323-32.

43. Zhang C, Basta T, Jensen ED, Klymkowsky MW. The beta-catenin/NegTregulated early zygotic gene Xnr5 is a direct target of SOX3 regulation. Development. 2003;130:5609-24

44. Wang Z, Oron E, Nelson B, Razis S, Ivanova N. Distinct lineage specification roles for NANOG, OCT4, and SOX2 in human embryonic stem cells. Cell Stem Cell. 2012;10:440-54

45. Abdelalim EM, Emara MM, Kolatkar PR. The SOX transcription factors as key players in pluripotent stem cells. Stem Cells Dev. 2014;23:2687-99.

46. Niwa H, Nakamura A, Urata M, Shirae-Kurabayashi M, Kuraku S, Russell S, et al. The evolutionally-conserved function of group B1 Sox family members confers the unique role of Sox2 in mouse ES cells. BMC Evol Biol. 2016;16:173.

47. Nakagawa M, Koyanagi M, Tanabe K, Takahashi K, Ichisaka T, Aoi T, et al. Generation of induced pluripotent stem cells without Myc from mouse and human fibroblasts. Nat Biotechnol. 2008;26:101-6. 
48. Yamaji M, Ueda J, Hayashi K, Ohta H, Yabuta Y, Kurimoto K, et al. PRDM14 ensures naive pluripotency through dual regulation of signaling and epigenetic pathways in mouse embryonic stem cells. Cell Stem Cell. 2013:12:368-82.

49. Takashima Y, Guo G, Loos R, Nichols J, Ficz G, Krueger F, et al. Resetting transcription factor control circuitry toward ground-state pluripotency in human. Cell. 2014;158:1254-69.

50. Niwa H, Ogawa K, Shimosato D, Adachi K. A parallel circuit of LIF signalling pathways maintains pluripotency of mouse ES cells. Nature. 2009;460:118-22.

51. Dalloul RA, Long JA, Zimin AV, Aslam L, Beal K, Blomberg Le A, et al. Multiplatform next-generation sequencing of the domestic Turkey (Meleagris gallopavo): genome assembly and analysis. PLoS Biol. 2010;8:e1000475.

52. Onichtchouk DV, Voronina AS. Regulation of zygotic genome and cellular pluripotency. Biochemistry (Mosc). 2015;80:1723-33.

53. Leichsenring M, Maes J, Mossner R, Driever W, Onichtchouk D. Pou5f1 transcription factor controls zygotic gene activation in vertebrates. Science. 2013;341:1005-9.

\section{Submit your next manuscript to BioMed Central} and we will help you at every step:

- We accept pre-submission inquiries

- Our selector tool helps you to find the most relevant journal

- We provide round the clock customer support

- Convenient online submission

- Thorough peer review

- Inclusion in PubMed and all major indexing services

- Maximum visibility for your research

Submit your manuscript at www.biomedcentral.com/submit 